\title{
Corrigendum: Risks for Child Cognitive Development in Rural Contexts
}

\begin{abstract}
Maria Julia Hermida ${ }^{1,2,3 *}$, Diego Edgar Shalom ${ }^{4,5}$, María Soledad Segretin 4,6, Andrea Paula Goldin ${ }^{1,4}$, Marcelo Claudio Abril ${ }^{2}$, Sebastián Javier Lipina ${ }^{4,6}$ and Mariano Sigman ${ }^{1,4,7}$
\end{abstract}

${ }^{1}$ Laboratorio de Neurociencia, Universidad Torcuato Di Tella, Buenos Aires, Argentina, ${ }^{2}$ Fundación Mundo Sano, Buenos Aires, Argentina, ${ }^{3}$ Instituto de Educación, Universidad Nacional de Hurlingham, Buenos Aires, Argentina, ${ }^{4}$ Consejo Nacional de Investigaciones Cientificas y Técnicas, Buenos Aires, Argentina, ${ }^{5}$ Departamento de Física, Facultad de Ciencias Exactas y Naturales, Universidad de Buenos Aires, Buenos Aires, Argentina, ${ }^{6}$ Unidad de Neurobiología Aplicada, Centro de Educación Médica e Investigaciones Clínicas "Norberto Quirno", Buenos Aires, Argentina, ${ }^{7}$ Facultad de Lenguas y Educación,

Universidad Nebrija, Madrid, Spain

Keywords: socioeconomic status, rural context, urban context, child cognitive development, executive functions, preschool attendance, father's educational level

\section{A Corrigendum on}

Risks for Child Cognitive Development in Rural Contexts by Hermida, M. J., Shalom, D. E., Segretin, M. S., Goldin, A. P., Abril, M. C., Lipina, S. J., et al. (2019). Front. Psychol. 9:2735. doi: 10.3389/fpsyg.2018.02735

In the original article, there were mistakes in Tables 2, 3, 4, and 6 as published.

There were three mistakes in Table 2. Firstly, column "Media" should be column "Mean." Secondly, in reference 6 (footnote) "subsides" should be "subsidies." Thirdly, in row "Frequency of mother child play (by week)," column "Urban/Mean" it says 2.14 and it should say 5.14.

There were two mistakes in Table 3. In row "Incubator/yes," column "Urban/valid percentage," it says 1.5 and it should say 10.5. Also, in row "Potential Nervous System conditions: No," column "Rural/ Valid percentage", it says 7.2 and it should say 70.2.

There were four mistakes in Table 4. Firstly, in rows " $0-3$ switchs," " $4-5$ switchs," and " 6 switchs," the word "switchs" should be "switches." Additionally, in row "Learning," column "Urban/mean," it says 2.87 and it should say 20.87 .

Finally, there were two mistakes in Table 6. In row "Number of siblings," column "F"' it says 2.151 and it should say 20.151. Also, in row "Past preschool attendance," column "F," it says 5.798 and it should say 50.798 .

The corrected Tables 2, 3, 4, and 6 appear below.

The authors apologize for these errors and state that they do not change the scientific conclusions of the article in any way. The original article has been updated.

\section{REFERENCES}

Hermida MJ, Shalom DE Segretin MS, Goldin AP, Abril MC, Lipina SJ and Sigman M (2021)

Corrigendum: Risks for Child

Cognitive Development in Rural Contexts. Front. Psychol. 12:647039. doi: $10.3389 /$ fpsyg.2021.647039
Instituto Nacional de Estadísticas y Censos (2010). Anuario Estadístico de la República Argentina. Buenos Aires: INDEC, 96.

Copyright (c) 2021 Hermida, Shalom, Segretin, Goldin, Abril, Lipina and Sigman. This is an open-access article distributed under the terms of the Creative Commons Attribution License (CC BY). The use, distribution or reproduction in other forums is permitted, provided the original author(s) and the copyright owner(s) are credited and that the original publication in this journal is cited, in accordance with accepted academic practice. No use, distribution or reproduction is permitted which does not comply with these terms. 
TABLE 2 | Descriptive statistic of the continuous variables obtained from parents' interviews in urban and rural contexts.

\begin{tabular}{|c|c|c|c|c|c|c|}
\hline \multirow[b]{2}{*}{ Continuous variables } & \multicolumn{3}{|c|}{ Urban } & \multicolumn{3}{|c|}{ Rural } \\
\hline & $n$ & Mean & $S D$ & $n$ & Mean & $S D$ \\
\hline Time of residence in the place & 83 & 4.88 & 2.28 & 48 & 5.88 & 0.61 \\
\hline Number of siblings ${ }^{1}$ & 83 & 1.34 & 0.99 & 47 & 2.53 & 1.36 \\
\hline Birth order ${ }^{2}$ & 82 & 1.76 & 0.90 & 47 & 2.62 & 1.21 \\
\hline Health history & 77 & 0.91 & 1.04 & 47 & 1.19 & 1.14 \\
\hline Pregnancy health history & 77 & 0.21 & 0.41 & 45 & 0.27 & 0.45 \\
\hline Father's age & 73 & 35.93 & 7.38 & 43 & 32.60 & 7.23 \\
\hline Mother's age & 76 & 32.89 & 6.93 & 48 & 29.21 & 7.15 \\
\hline Number of dependents in the household ${ }^{3}$ & 82 & 3.74 & 1.40 & 47 & 5.02 & 1.66 \\
\hline Father's completed level of education ${ }^{4}$ & 72 & 7.39 & 2.84 & 41 & 2.41 & 1.72 \\
\hline Mother's completed level of education & 79 & 7.22 & 3.30 & 43 & 2.93 & 2.31 \\
\hline Father's occupation ${ }^{5}$ & 78 & 3.73 & 1.79 & 45 & 1.51 & 1.01 \\
\hline Mother's occupation score & 81 & 2.16 & 2.44 & 46 & 0.33 & 0.97 \\
\hline Number of government subsidies ${ }^{6}$ & 77 & 0.51 & 0.62 & 47 & 1.02 & 0.49 \\
\hline Dwelling score & 77 & 11.26 & 1.47 & 47 & 8.62 & 2.13 \\
\hline Past preschool attendance ${ }^{7}$ & 77 & 21.97 & 9.62 & 47 & 7.91 & 5.75 \\
\hline Number of books at home & 77 & 1.95 & 1.00 & 47 & 2.81 & 0.54 \\
\hline Frequency of mother-child play (by week) & 76 & 5.14 & 2.40 & 47 & 4.62 & 2.85 \\
\hline Frequency of reading newspapers (by week) & 67 & 2.49 & 2.28 & 46 & 3.83 & 2.69 \\
\hline Frequency of watching TV (by week) & 73 & 5.66 & 2.14 & 47 & 4.83 & 3.04 \\
\hline Frequency of listening to the radio (by week) & 71 & 1.92 & 2.83 & 47 & 4.30 & 3.34 \\
\hline Frequency of using computers (by week) & 71 & 2.83 & 3.03 & 47 & 0.36 & 1.48 \\
\hline Frequency of using cellphone (by week) & 66 & 1.74 & 2.61 & 47 & 2.62 & 3.19 \\
\hline Mother anxiety & 67 & 8.91 & 3.54 & 37 & 9.08 & 3.93 \\
\hline Mother depression & 67 & 5.46 & 4.10 & 37 & 5.62 & 4.04 \\
\hline Surgency & 76 & 4.39 & 0.82 & 45 & 4.27 & 0.89 \\
\hline Negative affect & 76 & 4.60 & 0.79 & 45 & 4.37 & 0.85 \\
\hline Effortful control & 76 & 5.76 & 0.68 & 45 & 5.43 & 0.73 \\
\hline Age & 83 & 5.35 & 0.28 & 43 & 5.38 & 0.26 \\
\hline
\end{tabular}

${ }^{1}$ Total number of siblings of the child.

${ }^{2}$ Order in which the child was born.

${ }^{3}$ Number of people that economically depends on the household.

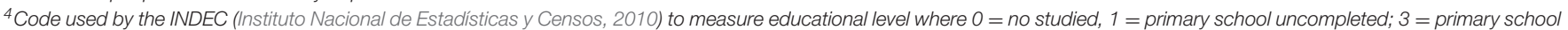
completed; 6 = high school uncompleted; 9 = high school completed/college uncompleted; 10 = college completed/graduate school uncompleted; 12 = graduate school completed.

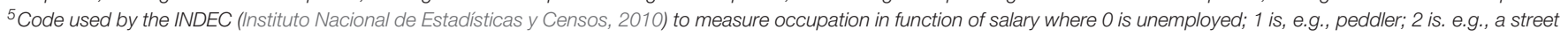
sweeper; 4 is, e.g., taxi driver; etc.

${ }^{6}$ Number of subsidies given by the government to that family.

${ }^{7}$ Amount of months that the child had attended school before the year of the study. 
TABLE 3 | Descriptive statistic of the nominal variables obtained from parents' interviews in urban and rural contexts.

\begin{tabular}{|c|c|c|c|c|c|}
\hline Nominal variables & \multicolumn{3}{|c|}{ Urban } & \multicolumn{2}{|c|}{ Rural } \\
\hline Gender & Boys & 46 & 55.4 & 22 & 44.6 \\
\hline \multirow[t]{2}{*}{ Parenting } & Father and mother & 62 & 74.7 & 40 & 83.3 \\
\hline & Mother & 15 & 18.1 & 6 & 12.5 \\
\hline \multirow[t]{2}{*}{ Low birth weight } & No & 66 & 91.7 & 32 & 88.9 \\
\hline & Yes & 6 & 8.3 & 4 & 11.1 \\
\hline \multirow[t]{2}{*}{ Preterm birth } & No & 68 & 75 & 36 & 81.8 \\
\hline & Yes & 8 & 16.7 & 8 & 18.2 \\
\hline Potential central nervous system conditions & No & 56 & 72.7 & 33 & 70.2 \\
\hline Hospital internship & Yes & 16 & 19.3 & 15 & 31.3 \\
\hline
\end{tabular}

TABLE 4 | Descriptive statistics of cognitive variables by context and UBN indicators.

\begin{tabular}{|c|c|c|c|c|c|c|}
\hline & \multicolumn{6}{|c|}{ Context } \\
\hline & $n$ & Mean & $S D$ & $n$ & Mean & $S D$ \\
\hline Attention & 41 & 5.34 & 3.60 & 79 & 7.11 & 3.88 \\
\hline Inhibitory control & 43 & 5.72 & 3.34 & 83 & 7.90 & 2.69 \\
\hline Learning & 44 & 14.05 & 9.54 & 82 & 20.87 & 9.79 \\
\hline Flexibility & & Frequencies & Percentage & & Frequencies & Percentage \\
\hline No switch & & 19 & 43.18 & & 14 & 17.50 \\
\hline 0-3 switches & & 1 & 2.27 & & 6 & 7.50 \\
\hline 4-5 switches & & 8 & 18.18 & & 5 & 6.25 \\
\hline
\end{tabular}

TABLE 6 | Significant results of ANOVAs: environmental and individual variables that differ by context.

\begin{tabular}{|c|c|c|c|c|}
\hline & $\boldsymbol{F}$ & $p$ & Non parametric control $p$-value & $\eta^{2}$ \\
\hline Number of siblings & 20.151 & 0.000 & 0.000 & 0.178 \\
\hline Birth order & 21.517 & 0.000 & 0.000 & 0.177 \\
\hline Father's completed level of education & 46.741 & 0.000 & 0.000 & 0.314 \\
\hline Father's occupation & 24.377 & 0.000 & 0.000 & 0.165 \\
\hline
\end{tabular}

